\title{
Microfluidic Construction of Hierarchically Composite Superballs for Sensing Applications ${ }^{+}$
}

\author{
Juan Wang ${ }^{1,2}$, Lingling Shui ${ }^{2, *}$, Loes I. Segerink ${ }^{1}$ and Jan C. T. Eijkel ${ }^{1, *}$ \\ 1 BIOS/Lab on a Chip Group, MESA+ Institute for Nanotechnology, University of Twente, Drienerlolaan 5, \\ 7522 NB Enschede, The Netherlands; Juan.wang@utwente.nl (J. W.); 1.i.segerink@utwente.nl (L.I.S.) \\ 2 National Center for International Research on Green Optoelectronics \& South China Academy of \\ Advanced Optoelectronics, South China Normal University, 510006 Guangzhou, China \\ * Correspondence: shuill@scnu.edu.cn (L.S.); j.c.t.eijkel@utwente.nl (J.C.T.E.); Tel.: +86-13437843083 (L.S.); \\ +31-534892839 (J.C.T.E.) \\ + Presented at the Eurosensors 2018 Conference, Graz, Austria, 9-12 September 2018.
}

Published: 30 November 2018

\begin{abstract}
Colloidal photonic crystals have attracted much attention of the scientific world due to their unique optical properties and potential applications in sensing, displays, optoelectronics, controlled superwetting and other fields. Here we report the fabrication of spherical colloidal photonic crystals featured with well-ordered nanopatterns from silica nanoparticles ( $\left.\mathrm{SiO}_{2} \mathrm{NPs}\right)$ and gold nanoparticles (AuNPs) through a droplet-based microfluidic approach. The colloidal crystals show both the photonic band gaps (PBG) and surface plasmonic resonance (SPR) properties. These proposed hierarchically composite 'superballs' will have an excellent performance in sensing applications, due to the fast response (the scattering color change) to the dielectric properties of the surrounding medium. A robust and efficient strategy is proposed and demonstrated to fabricate the composite superballs with multifunctional properties, broadening the perspective of their applications by the advantages of precise control over the size of the particles and flexible change of the fluid composition.
\end{abstract}

Keywords: photonic crystal; photonic band gaps; surface plasmonic resonance; composite superball

\section{Introduction}

Hierarchical architectures have been assembled from a variety of building blocks with different materials and functionalities. Particularly, photonic crystals are of great significance as particulate systems due to diverse applications including displays, biosensors, optoelectronic devices, catalysts, and controlled superwetting [1,2]. Light incident on the nanoparticles (of a size smaller than the wavelength) induces the conduction electrons in them to oscillate collectively with a resonant frequency that depends on the nanoparticles' size, shape, composition and the local dielectric environment [3]. These noble metal nanoparticles can serve as extremely intensive and stable labels for bio-/chemical-sensors, surface-enhanced spectroscopy and immunoassays [4]. Researchers have been investigating the fabrication of composite particles by incorporation of these metal nanoparticles with non-metallic nanoparticles $\left(\mathrm{SiO}_{2} \mathrm{NPs}\right)$, polystyrene nanoparticles etc.), combining each particle's own specific optical properties in order to broaden the applications. Tadashi Nakamura and their colleagues [5] have prepared AuNPs-embedded nanoporous spheres and the corresponding closepacked colloidal crystal array. This 2D array exhibited both the optical stop band from the Bragg diffraction and the surface plasmon resonance (SPR) from the gold. Frank Caruso and his colleagues [6] have prepared core-shell and hollow colloid spheres derived from $\mathrm{Au} @ \mathrm{SiO}_{2}$ nanoparticles by using the layer-by-layer self-assembly technique. AuNPs of $\sim 5 \mathrm{~nm}$ decorating the surface of silica submicrospheres were fabricated by ultrasound with the advantage of simple and efficient 
production [7]. Most of the previous work has focused on the fabrication of satellite-like composite particles, or particles which served as new building blocks to construct 2D photonic crystals with SPR properties.

However, hierarchically composite superballs with both photonic band gap (PBG) and SPR properties have not been proposed before, and we verify for the first time through their production by droplet microfluidic techniques, incorporating the AuNPs and $\mathrm{SiO}_{2} \mathrm{NPs}$ as building blocks. The particles can be used as a sensing unit resulting from the scattering color response to a change of the dielectric properties of the surroundings.

\section{Materials and Methods}

\subsection{Materials}

$\mathrm{N}$-isopropylacrylamide (NIPAM, purity $\geq 99 \%$ ) and N, N-methylene bisacrylamide (MBA, purity $\geq 99 \%$ ) were purchased from Sigma Aldrich (Zwijndrecht, The Netherlands). 2, 2Diethoxyacetophenone (DEAP, purity $\geq 98 \%$ ) was purchased from Sigma Aldrich (Zwijndrecht, The Netherlands). Hexadecane and Sorbitane monooleate (Span80) were purchased from Sigma Aldrich (Zwijndrecht, The Netherlands). AuNPs were purchased from Cytodiagnostics Inc. company (Burlington, ON, Canada). $\mathrm{SiO}_{2} \mathrm{NPs}$ were all purchased from Nanjing Nanorainbow Biotechnology Co. Ltd. company (Nanjing, China). Absolute ethanol solution (analysis level) was purchased from Merck KGaA (Zwijndrecht, The Netherlands).

\subsection{Preparation of 3D Spherical Colloidal Photonic Crystals}

A flow-focusing droplet microfluidic chip made by PDMS was used to generate the hybrid nanoparticles laden droplets with a good uniformity and high stability. The oil phase was hexadecane with $18 \mathrm{wt} \%$ Span 80 as the surfactant to stabilize the generated droplets and $5 \mathrm{wt} \%$ DEAP served as the photoinitiator. The aqueous suspension was composed of $\mathrm{SiO}_{2} \mathrm{NPs}$ and AuNPs and also contained water-soluble monomer and cross-linker. Monodispersed droplets were sheared off by the oil phase at the flow-focus junction. The nanoparticles confined in these produced droplets were selfassembled due to the electrostatic repulsion. Solid 3D spherical photonic crystals were yielded upon UV irradiation (IntelliRay-600, $365 \mathrm{~nm}, 600 \mathrm{~W}, 120 \mathrm{~mW} / \mathrm{cm}$, Uvitron International, Inc., West Springfield, MA, USA). In this method, the size and ratio of the building blocks ( $\mathrm{SiO}_{2} \mathrm{NPs}$ and AuNPs) can be precisely and flexibly tuned to produce the 3D photonic crystals with SPR properties, which was attributed to the advantages of the microfluidic approach.

\section{Results and Discussion}

3D composite superballs with both PBG and SPR properties, dependent on the size and shape of the building blocks and surrounding media, respectively, have been produced based by a robust and efficient strategy.

\subsection{Fabrication of 3D Composite Superballs}

Monodispersed droplets encapsulating $\mathrm{SiO}_{2} \mathrm{NPs}$ and AuNPs are generated by the droplet microfluidic platform. The schematic Figure 1a, shows the generation procedure. Water-soluble fluids containing monomer of 5.5\% NIPAM, cross-linker of $2 \%$ MBA as well as $\mathrm{SiO}_{2} \mathrm{NPs}$ in $100 \mathrm{~nm}$ and AuNPs with a varying diameter, were used as the dispersed phase, which was sheared off by the continous phase to form droplets. Figure $1 \mathrm{~b}$ shows the optical microscopy image of the droplet generation at the flow-focus junction. Furthermore, the size and the ratio of the composite building blocks were precisely tuned to produce hierarchically composed superballs, showing a variety of PBG and SPR properties (Figure 2). 


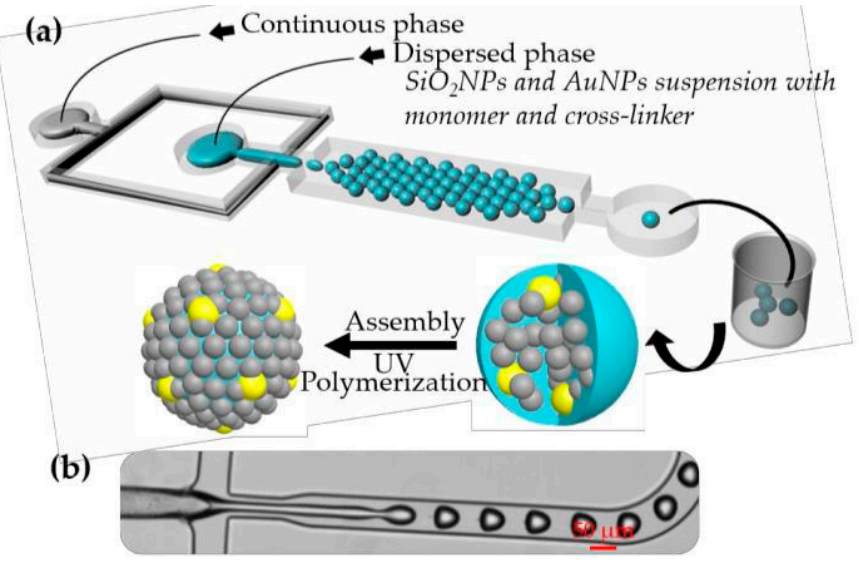

Figure 1. (a) Schematic of the composite superball fabrication process by droplet microfluidic platform; (b) Optical image of droplet generation.
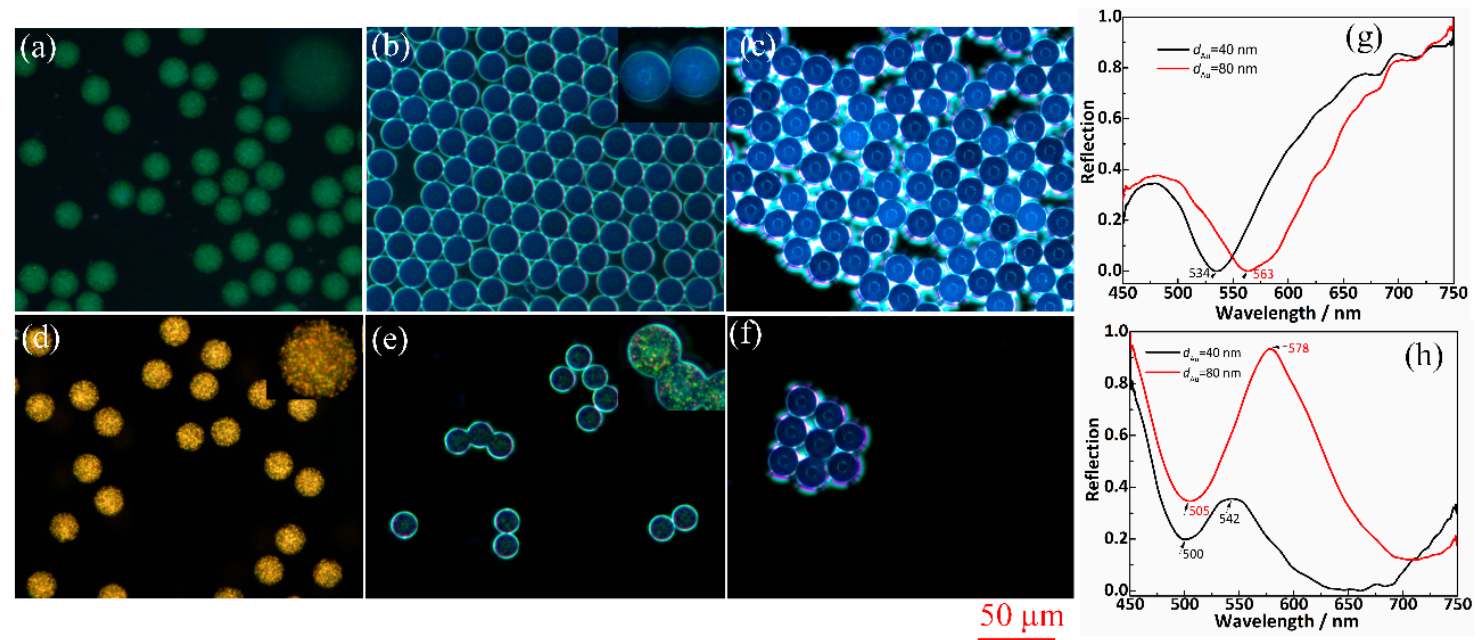

Figure 2. Optical images of the superball scattering color change in response to the surrounding medium. Superballs were made from $d_{\mathrm{AuNPs}}=40 \mathrm{~nm}$ and $d_{\mathrm{SiO} \mathrm{NPs}_{\mathrm{s}}}=100 \mathrm{~nm}(\mathbf{a}-\mathbf{c})$ and $d_{\mathrm{AuNPs}}=80 \mathrm{~nm}$ and $d \mathrm{SiO}_{2} \mathrm{NPs}_{\mathrm{s}}=100 \mathrm{~nm}$. $(\mathbf{d}-\mathbf{f})$ The superballs were suspended in $(\mathbf{a}, \mathbf{d})$ hexadecane, $(\mathbf{b}, \mathbf{e})$ deionized water, $(\mathbf{c}, \mathbf{f})$ air. $(\mathbf{g}, \mathbf{h})$ show the reflection spectra of the superballs in hexadecane ('oil') under the bright field and dark filed of optical microscope, respectively.

\subsection{Controllable PBG and SPR Properties}

It has been demonstrated that the scattering color of these superballs can be tuned by the size of $\mathrm{SiO}_{2} \mathrm{NPs}$ and AuNPs, the ratio of $\mathrm{SiO}_{2} \mathrm{NPs}$ and AuNPs, as well as the surrounding medium. Figure 2 illustrates the scattering color change in response to the AuNP diameter and medium refractive index. The scattering color resulted from the SPR property of the AuNPs integrated into the superballs when they were suspended in solution. However, in the dry state exposed to air, the observed scattering color was caused by the photonic crystal properties (PBG) of the periodic nanostructure from the close-packed $\mathrm{SiO}_{2} \mathrm{NPs}$. Figure 3 showed the scanning electronic microscope (SEM) images of the superball morphologies corresponding to Figure 2. 

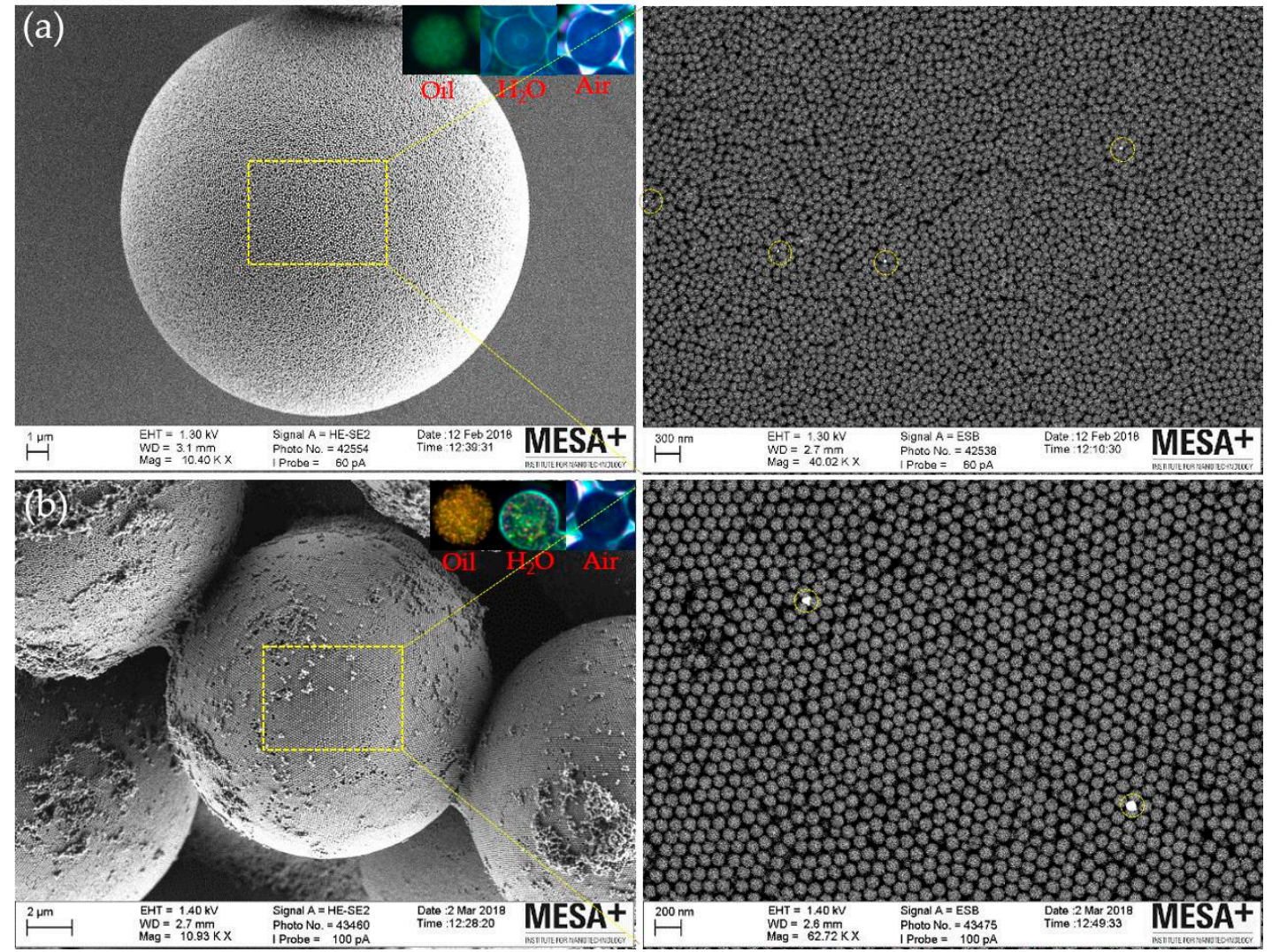

Figure 3. SEM images of composite superballs made from (a) $\mathrm{SiO}_{2} \mathrm{NPs}_{\mathrm{s}}(100 \mathrm{~nm})$ and AuNPs (40 nm), and (b) $\mathrm{SiO}_{2}$ NPs $(100 \mathrm{~nm})$ and AuNPs $(80 \mathrm{~nm})$. The AuNPs are visible inside the yellow circles.

\section{Conclusions}

3D hierarchically composed superballs self-assembled from $\mathrm{SiO}_{2} \mathrm{NPs}$ and AuNPs have been proposed and their production was verified via a microfluidic technique, showing both PBG and SPR properties. This proposed strategy shows advantages of facile and fast fabrication, and offers opportunities of integration of PBG and SPR properties into one micro-unit. The combination would have high potential for biosensor applications, as the sensitivity to the refractive index of the environment can for example be exploited to probe antigen-antibody reactions or nucleic acid hybridization.

Acknowledgments: This work was supported by Oversea Study Program of Guangzhou Elite Project and a Pioneers in Healthcare voucher (project Ischemia on chip) of the University of Twente, MST and ZGT in the Netherlands.

Conflicts of Interest: There are no conflicts to declare.

\section{References}

1. Li, H.; Wang, J.; Yang, L.; Song, Y. Superoleophilic and superhydrophobic inverse opals for oil sensors. Adv. Funct. Mater. 2008, 18, 3258-3264.

2. Yin, S.N.; Wang, C.F.; Liu, S.S.; Chen, S. Facile fabrication of tunable colloidal photonic crystal hydrogel supraballs toward a colorimetric humidity sensor. J. Mater. Chem. C 2013, 1, 4685-4690.

3. Jensen, T.R.; Malinsky, M.D.; Haynes, C.L.; Van Duyne, R.P. Nanosphere lithography: Tunable localized surface plasmon resonance spectra of silver nanoparticles. J. Phys. Chem. B 2000, 104, 10549-10556.

4. Chen, Y.; Munechika, K.; Ginger, D.S. Dependence of fluorescence intensity on the spectral overlap between fluorophores and plasmon resonant single silver nanoparticles. Nano Lett. 2007, 7, 690-696.

5. Nakamura, T.; Yamada, Y.; Yano, K. Direct synthesis of monodispersed thiol-functionalized nanoporous silica spheres and their application to a colloidal crystal embedded with gold nanoparticles. J. Mater. Chem. 2007, 17, 3726-3732. 
6. Caruso, F.; Spasova, M.; Salgueiriño-Maceira, V.; Liz-Marzán, L.M. Multilayer assemblies of silicaencapsulated gold nanoparticles on decomposable colloid templates. Adv. Mater. 2001, 13, 1090-1094.

7. Pol, V.; Gedanken, A.; Calderon-Moreno, J. Deposition of gold nanoparticles on silica spheres: A sonochemical approach. Chem. Mater. 2003, 15, 1111-1118.

(C) 2018 by the authors. Licensee MDPI, Basel, Switzerland. This article is an open access article distributed under the terms and conditions of the Creative Commons Attribution (CC BY) license (http://creativecommons.org/licenses/by/4.0/). 